\title{
NOTE ON THE RE-TOPOLOGIZATION OF A SPACE BY A SET OF OPERATORS
}

\author{
YOSHIO ITAGAKI
}

(Received November 6, 1968)

Let $\left\{T_{i}\right\} \quad(i \in A)$ be a set of linear operators in a locally convex vector space $E$. In [2], M. Nagumo tried to adopt a new topology on $E$, which makes each $T_{i}$ be continuous and makes it possible to extend $T_{i}$ on all elements of $E$. His method is a generalization of a so-called Lax's negative norms. As the special cases of it we get Schwartz's distribution space and others. The purpose of this paper is to weaken the condition on $E$ supposed by M. Nagumo. It may be said that our proof corresponds naturally to the technique used in the extension of differential operators.

As J. E. Roberts noted in [4], in particular taking a countable set of closed operators in a Hilbert space, we meet with a countably Hilbert space in the sense of Gel'fand [1]. We note here that the converse is also true, more precisely, that the topology of any countably Hilkert space can be constructed as the projective limit by a set of self-adjoint operators in a Hilbert space.

Let $E$ be a separated locally convex vector space and $\left\{T_{i}\right\} \quad(i \in A)$ be a set of linear operators from $E$ to $E$. We assume that $D\left(T_{i}\right)$, the domain of each $T_{i}$, is dense in $E$ and that the set $\left\{T_{i}\right\}$ contains identity operator $I$. We shall from now onwards take the set of operators satisfying the following condition:

(C). $F=\bigcap_{i \in A} D\left(T_{i}{ }^{\prime}\right)$ is total over $E$, i.e., the element $x$ of $E$, for which $\langle f, x\rangle=0$ for all $f$ of $F$, is zero element. Here by $T_{i}^{\prime}$ we denote the transpose of $T_{i}$.

If the set $D\left(T_{i}{ }^{\prime}\right)$ is total over $E, T_{i}$ is a pre-closed operator. Therefore when the above condition is satisfied, all $T_{i}$ are pre-closed. For the reflexive space $E$ condition (C) means that $F$ is dense in $E^{\prime}$, the dual space of $E$. Here the dual space of $E$ is considered under the strong topology $\beta\left(E^{\prime}, E\right)$. We shall denote it by $E_{\beta}^{\prime}$ later.

Our main problem is to introduce a suitable topology in $E$ and to make any $T_{i}$ continuous from $D_{i}=D\left(T_{i}\right)$ to $E$. In that case, space $E$ under such a topology we denote by $\widehat{E}$, and write completion of $\widehat{E}$ by $\widetilde{E}$. Then, under the 
some condition on $\left\{T_{i}\right\}, T_{i}$ can be uniquely extended to continuous operators on $\widetilde{E}$.

THEOREM 1. Suppose that $E$ is a quasi-t-space, i.e., every strongly bounded set in $E_{\beta}^{\prime}$ is equi-continuous. Then, under the condition (C), we can give a new topology to $E$, in such a way that all $T_{i}$ will be continuous from $E$ with the primary topology into $\widehat{E}, E$ with new topology.

We remark here that bornological or barreled space is quasi-t-space. M. Nagumo proved this theorem for the bornological space.

ProOF. Since $E$ is a quasi-t-space, natural embedding $\pi$ from $E$ to $E^{\prime \prime}=\left(E_{\beta}^{\prime}\right)_{\beta}^{\prime}$, the bidual of $E$, is a topological isomorphism [5, p.71]. We introduce on $F$ the coarsest convex topology which makes every $T_{i}^{\prime}$ to be a continuous operator from $F$ to $E_{3}^{\prime}$, and write $F$ with this topology by $\widehat{F}$. That is, the topology on $F$ is the projective topology with respect to $\left\{\left\{E_{\beta}^{\prime}, T_{i}^{\prime}\right\} i \in A\right\}[5$, p. 84]. By the assumption that $\left\{T_{i}\right\}$ contains $I$, the topology of $\widehat{F}$ is finer than that of $E_{\beta}^{\prime}$ and so $\widehat{F}$ is separated. Any $T_{i}^{\prime \prime}$, the transpose of $T_{i}^{\prime}$, the operator from $E^{\prime \prime}$ to $\widehat{F}_{\beta}^{\prime}$, is strongly continuous. We restrict the domain of $T_{i}^{\prime \prime}$ to $\pi\left(D_{i}\right)$. When we identify $\pi\left(D_{i}\right)$ to $D_{i}, T_{i}^{\prime \prime}$ and $T_{i}$ become algebraically equivalent. This fact is assured by that $F$ is total over $E$. The space $E$, considered as a subspace of $\widehat{F}_{\beta}^{\prime}$, is denoted by $\widehat{E}$. This space satisfies the condition of the theorem.

THEOREM 2. In Theorem 1, let $\widehat{D_{i}}$ be the space $D_{i}$ with relative topology induced by $\widehat{E}$, and assume that for any $i, j \in A$ there exists $k \in A$ satisfying $T_{j}\left(T_{i}\right) x=T_{k} x$ for any $x$ of $D_{i}$. Then any $T_{i}$ is a continuous operator from $\widehat{D_{i}}$ to $\widehat{E}$, therefore uniquely can be extended to a continuous operator on $\widetilde{E}$, which denotes the completion of $\widehat{E}$.

PROOF. As $\widehat{F}$ has the projective limit topology with respect to the mappings $\left\{\left\{E_{\beta}^{\prime}, T_{i}^{\prime}\right\} i \in A\right\}, T_{i}^{\prime}: \widehat{F} \rightarrow \widehat{F}$ are continuous if and only if $T^{\prime}, T_{i}^{\prime}: \widehat{F} \rightarrow \widehat{E}_{\beta}^{\prime}$ are continuous for all $j \in A[5, \mathrm{p}$.84]. This latter condition is satisfied by the hypothesis. Thus all $T_{i}^{\prime}$ are continuous from $\widehat{F}$ to $\widehat{F}$, so that all $T_{i}^{\prime \prime}$ are also continuous, a fortiori $T_{i}^{\prime \prime}$ restricted to $\widehat{D}_{i}$.

The validity of last part of the theorem follows from the fact that $D_{i}$ is dense in $E$ and $E$ is dense in $\widetilde{E}$, so $\widehat{D}_{i}$ dense in $\widetilde{E}$.

THEOREM 3. If $\widehat{F}$ is reflexive and $\widehat{F}_{\beta}^{\prime}$ is complete, $\widetilde{E}$ is the dual space 
of $\widehat{F}$.

PROOF. $\widehat{E}$ is a linear subspace of $\widehat{F}_{\beta}^{\prime}$, and by assumption $\widetilde{E}$ is also a subspace of $\widehat{F}_{\beta}^{\prime}$. If $\widetilde{E} \neq \widehat{F}_{\beta}^{\prime}$, there exists $f$, a non-zero element of $\left(\widehat{F}_{\beta}^{\prime}\right)^{\prime}=\widehat{F}$, such that $\langle f, x\rangle=0$ for all $x$ of $\widetilde{E}$. But this is impossible since $f \in \widehat{F} \subset E^{\prime}$ and $E \subset \widetilde{E}$.

It must be remarked that if $\widehat{F}$ is bornological, its dual space $\widehat{F}_{\beta}^{\prime}$ is complete [5, p. 104]. Fréchet space is bornological, and Fréchet space very well arises in application in the following form [3].

THEOREM 4. Let $E$ be a reflexive Banach space and $A$ be a countable set. Then $\widehat{F}$ constructed above is a reflexive Frèchet space.

Proof. As the transpose of every $T_{i}$ is a closed operator and $\left\{T_{i}\right\}$ contains $I, \widehat{F}$ is isomorphic to a closed subspace of the product $\prod_{i \in A} E_{i}^{\prime}$, where $E_{i}^{\prime}=E_{\beta}^{\prime}[5$, p. 88]. Since $E_{\beta}^{\prime}$ is a reflexive Banach space, $\prod_{i \in A} E_{i}^{\prime}$ is reflexive and therefore $\widehat{F}$ is also reflexive Fréchet space.

Acknowledgement. I wish thank Professor M. Fukamiya for useful suggestions and Professor J. E. Roberts who kindly corrected errors in a previous version.

\section{REFERENCES}

[1] I. M. GeL'FAND AND N. YA. VILENKIN, Generalized Functions, Vol. 4, (English translation) Academic Press, 1964.

[2] M. NAGUMO, Re-topologization of functional space in order that a set of operators will be continuous, Proc. Japan Acad., 37(1961), 550-552.

[ 3 ] M. NAGUMO, Re-topologization for continuity of a set of operators, Sûgaku, 14-3(1963), 164-166.

[ 4 ] J. E. RoBER's, Rigged Hilbert spaces in quantum mechanics, Comm. Math. Phys., 3(1966), 98-119.

[ 5 ] A. P. and W. J. Robertson, Topological Vector Spaces, Cambridge, 1967.

DEPARTMENT OF MATHEMATICS

MIYAGI UNIVERSITY OF EDUCATION

SENDAI, JAPAN 